Canad. Math. Bull. Vol. 23 (2), 1980

\title{
RETRACTS AND THE FIXED POINT PROBLEM FOR FINITE PARTIALLY ORDERED SETS
}

\author{
BY \\ DWIGHT DUFFUS, WERNER POGUNTKE AND IVAN RIVAL
}

A partially ordered set $P$ has the fixed point property if every orderpreserving mapping $f$ of $P$ to $P$ has a fixed point, that is, $f(a)=a$ for some $a \in P$; call $P$ fixed point free if $P$ does not have the fixed point property.

Problem.

Characterize those partially ordered

sets with the fixed point property.

For those partially ordered sets that are lattices the solution is, of course, provided by the beautiful result of Tarski [9] and Davis [3]:

Let $L$ be a lattice. Then $L$ has the fixed point property if and only if $L$ is $a$ complete lattice.

In short, a lattice $L$ that is not complete is fixed point free because it contains a chain $C$ that is itself fixed point free and, there exists an orderpreserving mapping $f$ of $L$ to $L$ such that $f(L)=C$ and $f \mid C$ is the identity mapping of $C$. Since there is an order-preserving mapping $g$ of $C$ to $C$ that is fixed point free, $g \circ f$ is a fixed point free mapping of $L$ to $L$ [3].

Apart from this result, little is known.

We call a subset $Q$ of a partially ordered set $P$ a retract of $P$ if there is an order-preserving mapping $f$ of $P$ to $P$ such that $f(P)=Q$ and $f \mid Q$ is the identity mapping of $Q$; the mapping $f$ we call a retraction mapping of $P$ onto $Q$. For instance, a lattice that is not complete contains a fixed point free chain as a retract. In fact, it is shown in [6] that each maximal chain in a partially ordered set $P$ is a retract of $P$. It follows that if $P$ has the fixed point property then every maximal chain in $P$ is complete. Of course, the converse of this statement is far from true-every chain in a finite, fixed point free partially ordered set is complete.

It is easy to see that a partially ordered set $P$ has the fixed point property if and only if every retract of $\boldsymbol{P}$ has the fixed point property. We can say somewhat more for finite partially ordered sets.

Proposition 1. A finite partially ordered set $P$ is fixed point free if and only if there is a retract $Q$ of $P$ with a fixed point free automorphism.

Received by the editors January 25, 1978 and in revised form, September 25, 1978 and January 29, 1979. 
Proof. If $g$ is a retraction mapping of $P$ onto $Q$ and $h$ is a fixed point free automorphism of $Q$ then $h \circ g$ is a fixed point free mapping of $P$ to $P$.

Conversely, let $f$ be a fixed point free mapping of $P$ to $P$. Since $P$ is finite there is a positive integer $n$ such that $f^{n}(P)=f^{n+1}(P)$, where $f^{1}=f$ and $f^{i+1}=f \circ f^{i}$. Then $f^{\prime}=f \mid f^{n}(P)$ is an automorphism of $f^{n}(P)$. Let $Q=f^{n}(P)$. There is a positive integer $k$ such that $\left(f^{\prime}\right)^{k}$ is the identity mapping of $Q$. It follows that $f^{n k}(P)=Q$ and $f^{n k} \mid Q$ is the identity mapping of $Q$. In other words, $Q$ is a retract of $P$ and $f^{\prime}$ is a fixed point free automorphism of $Q$.

Proposition 1 is a useful tool in studying the fixed point property for finite partially ordered sets. As an example we shall prove a result providing a sufficient condition for the fixed point property. First we require some terminology and another fact concerning retracts of finite partially ordered sets.

Let $P$ be a partially ordered set containing no infinite chains and let $\max (P)$ $(\min (P))$ denote the set of maximal (minimal) elements of $P$. Call $S \subseteq P$ a spanning subset of $P$ if $\max (S) \cup \min (S) \subseteq \max (P) \cup \min (P)$. Let $Q$ be a retract of $P$ and suppose $x \in \max (Q)-\max (P)$. Now choose $x^{\prime} \in \max (P), x<x^{\prime}$. If $y \in Q$ and $y<x^{\prime}$ then $y \leq x$. Therefore, $Q^{\prime}=(Q-\{x\}) \cup\left\{x^{\prime}\right\} \cong Q$ and $Q^{\prime}$ is also a retract of $P$-if $f$ is a retraction mapping of $P$ onto $Q$ then $f^{\prime}$, defined by

$$
f^{\prime}(z)=\left\{\begin{array}{cl}
f(z) & \text { if } f(z) \neq x \\
x^{\prime} & \text { if } f(z)=x,
\end{array}\right.
$$

is a retraction mapping of $P$ onto $Q^{\prime}$. We summarize: Let $P$ be a partially ordered set containing no infinite chains and let $Q$ be a retract of $P$. Then there is a spanning subset $Q^{\prime}$ of $P$ such that $Q^{\prime} \cong Q$ and $Q^{\prime}$ is a retract of $P$.

For $S \subseteq P$ let $S_{*}=\{x \in P \mid x \leq s$ for every $s \in S\}$.

THEOREM 2. Let $P$ be a finite partially ordered set and let $S_{*}$ have the fixed point property for every nonempty subset $S$ of $\max (P)$. Then $P$ has the fixed point property.

Proof. Let us suppose that $P$ is fixed point free. By Proposition 1 there is a retraction mapping $f$ of $P$ onto a spanning subset $Q$ of $P$; moreover, $Q$ has a fixed point free automorphism $g$. Let $b \in \max (Q) \subseteq \max (P)$ and let $B=$ $\left\{b, g(b), g^{2}(b), \ldots\right\}$. Since $g$ is an automorphism of $Q, B \subseteq \max (Q) \subseteq \max (P)$ : we claim that $B_{*}$ is fixed point free.

As the empty set is (trivially) fixed point free, we may assume that $B_{*} \neq \phi$. Let $x \in B_{*}$. Then $x \leq g^{i}(b)$ for $i=0,1, \ldots$ (taking $\left.g^{0}(b)=b\right)$, so $f(x) \leq$ $f\left(g^{i}(b)\right)=g^{i}(b)$ for $i=0,1, \ldots$, and $f\left(B_{*}\right) \subseteq B_{*} \cap Q$. Now, if $y \in B_{*} \cap Q$ then $g(y) \leq g^{i}(b)$ for $i=0,1, \ldots$; hence, $g\left(B_{*} \cap Q\right) \subseteq B_{*} \cap Q$. We have shown that $f\left(B_{*}\right)=B_{*} \cap Q, f \mid B_{*} \cap Q$ is the identity on $B_{*} \cap Q-B_{*} \cap Q$ is a retract of $B_{*}$-and $g \mid B_{*} \cap Q$ is a fixed point free automorphism. Therefore, $g \circ f \mid B_{*}$ is a fixed point free mapping of $B_{*}$. 


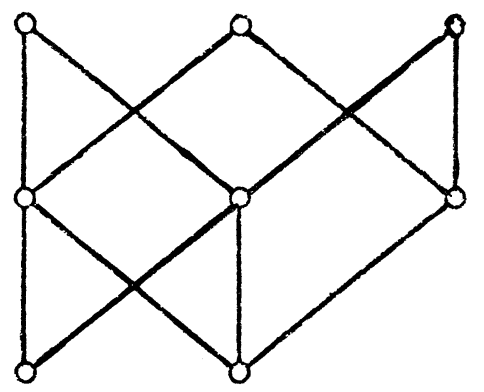

Figure 1

In [7], H. Höft and M. Höft prove that a partially ordered set $P$ has the fixed point property if every maximal chain of $P$ is a complete sublattice and $P$ contains only finitely many maximal elements every nonempty subset of which has an infimum. As any finite partially ordered set with a maximum element has the fixed point property, Theorem 2 sharpens the finite version of this result of Höft and Höft. The converse of Theorem 2, of course, fails (see Figure 1).

Theorem 2 is inspired by a question communicated to us by J. R. Isbell who attributes it to L. Mohler. Let $P$ be a finite partially ordered set, let $\max (P)=$ $A \cup B$, where $A \cap B=\phi$, and for $S \subseteq P$, set $S^{\prime}=\{x \in P \mid x \leq s$ for some $s \in S\}$. The question: Does $P$ have the fixed point property if each of $A^{\prime}, B^{\prime}$, and $A^{\prime} \cap B^{\prime}$ has the fixed point property? While Theorem 2 provides a positive answer to this question in the special case that $|\max (P)| \leq 2, \mathrm{~K}$. Baclawski and $\mathrm{A}$. Björner in [1] report an example that provides a negative answer to the general question.

A subset $Q$ of a partially ordered set $P$ is a fixed point set of $P$ if there is an order-preserving mapping $f$ of $P$ to $P$ such that $Q=\{x \in P \mid f(x)=x\}$.

Problem. Characterize those subsets of a partially ordered set that are fixed point sets.

Certainly a retract of $P$ is a fixed point set of $P$. Nonetheless, a fixed point set need not be a retract (see Figure 2).

Again, for complete lattices the answer is at hand. Let $L$ be a complete lattice and let $K$ be a fixed point set of $L$. It is well-known that $K$ (with the induced partial ordering) is a lattice; in fact, $K$ is a complete lattice. It is an easy matter to show that any subset $Q$ of a partially ordered set $P$ is a retract of $P$ provided that $Q$ is a complete lattice. (This fact is implicit in G. Birkhoff [2, pp. 301-302].) Combining these facts yields a description of fixed point sets 


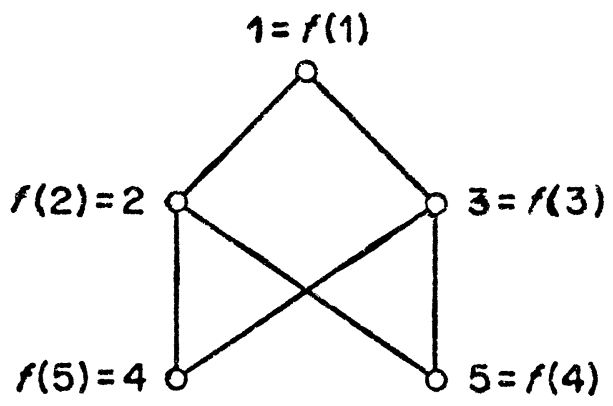

Figure 2

of complete lattices: If $L$ is a complete lattice and $K$ is a subset of $L$ then $K$ is $a$ fixed point set of $L$ if and only if $K$ is a complete lattice.

For an integer $n \geq 3$, a crown is a partially ordered set $\left\{x_{1}, y_{1}, x_{2}, y_{2}, \ldots, x_{n}\right.$, $\left.y_{n}\right\}$ in which $x_{i} \leq y_{i}, x_{i+1} \leq y_{i}$, for $i=1,2, \ldots, n-1, x_{1} \leq y_{n}$ and $x_{n} \leq y_{n}$ are the only comparability relations (see Figure 3 ). A four-crown in a partially ordered set $P$ is a set $\left\{x_{1}, y_{1}, x_{2}, y_{2}\right\}$ such that $x_{i}<y_{j}$, for $i, j=1,2$, are the only comparabilities and there is no $z \in P$ such that $x_{1}, x_{2} \leq z \leq y_{1}, y_{2}$. D. Duffus and I. Rival [5] have shown that if $P$ is a finite, connected partially ordered set containing no crowns then a subset $Q$ of $P$ is a fixed point set of $P$ if and only if $Q$ is a retract of $P$.

Let $\boldsymbol{P}$ be a finite partially ordered set. For elements $a>b$ in $P$, $a$ covers $b$ $(a>b)$ if, for all $c \in P, a \geq c>b$ implies $a=c ; a$ is irreducible in $P$ if $a$ has precisely one upper cover or precisely one lower cover in $P$. Let $I(P)$ denote the set of irreducible elements of $P$. Note that $P$ has the fixed point property if and only if $P-\{a\}$ has the fixed point property for all $a \in I(P)[8] . P$ is dismantlable (by irreducibles) if $P=\left\{a_{1}, a_{2}, \ldots, a_{n}\right\}$ and

$$
a_{i} \in I\left(P-\left\{a_{1}, a_{2}, \ldots, a_{i-1}\right\}\right)
$$

for $i=1,2, \ldots, n-1$. A dismantlable partially ordered set has the fixed point property [8].

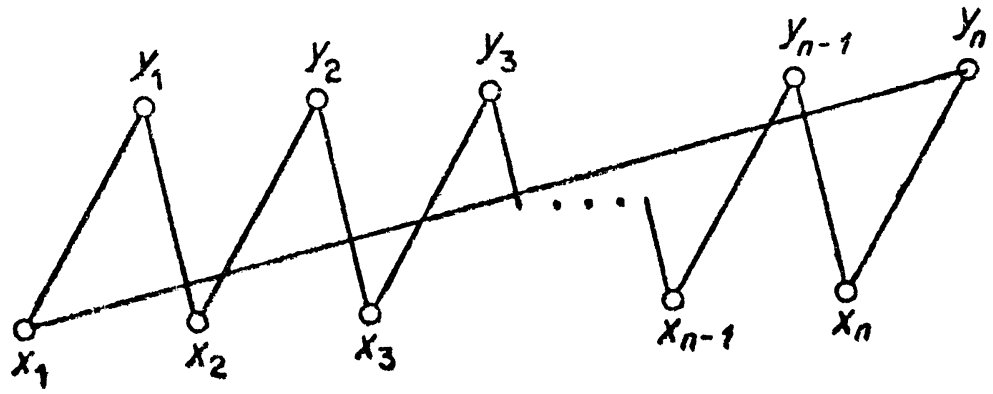

Figure 3 
A finite, connected partially ordered set containing no crowns is dismantlable [4]. Still, not every fixed point set of a dismantlable partially ordered set is a retract (see Figure 2).

THEOREM 3. Let $P$ be a dismantlable partially ordered set and let $Q$ be a fixed point set of $P$. Then $Q$ is dismantlable.

The proof of Theorem 3 is based on two lemmas.

LemMa 4 [4]. Let $P$ be a dismantlable partially ordered set and let $a \in I(P)$. Then $P-\{a\}$ is dismantlable.

LEMMA 5. Let $P$ be a dismantlable partially ordered set and let $Q$ be a retract of $P$. Then $Q$ is dismantlable.

Proof. We proceed by induction on $|P|$. Let $f$ be a retraction mapping of $P$ onto $Q$. Let $a \in I(P)$. Since $P$ is dismantlable, $P-\{a\}$ is dismantlable.

If $a \in I(P)-Q$ then $Q$ is a retract of $P-\{a\}$, whence, by the induction hypothesis, $Q$ is dismantlable. If $a \in I(P) \cap I(Q)$ then, with $a^{*}$ as the unique upper cover of $a$ in $Q$, define $f^{\prime}$ of $P-\{a\}$ to $Q-\{a\}$ by

$$
f^{\prime}(z)= \begin{cases}f(z) & \text { if } f(z) \neq a \\ a^{*} & \text { if } f(z)=a .\end{cases}
$$

Then $f^{\prime}$ is a retraction mapping of $P-\{a\}$ onto $Q-\{a\}$. Again, the induction hypothesis implies that $Q$ is dismantlable.

Let $a \in I(P)$, let $a \in Q-I(Q)$, and let $a^{*}$ be the unique upper cover of $a$ in $P$. Since $a \notin I(Q), a^{*} \notin Q$. Since $a^{*} \geq a, f\left(a^{*}\right) \geq f(a)=a$. If $f\left(a^{*}\right)>a$ then $a$ is not maximal in $Q$ so there are distinct elements $b, c$ in $Q$ such that $b>a$ and $c>a$ in $Q$; hence $b \geq a^{*}$ and $c \geq a^{*}$ in $P$, so $b=f(b) \geq f\left(a^{*}\right)$ and $c=f(c) \geq f\left(a^{*}\right)$. Therefore, $f\left(a^{*}\right)=a$. We claim that $Q^{\prime}=(Q-\{a\}) \cup\left\{a^{*}\right\} \cong Q$. Let $x \in Q-\{a\}$. Then $a<x$ implies $a<a^{*}<x, x<a$ implies $x<a^{*}$, and $a^{*}<x$ implies $a<x$. Let $x<a^{*}$. Then $x=f(x) \leq f\left(a^{*}\right)=a$. Hence, $Q \cong Q^{\prime}$.

Since $Q^{\prime}$ is contained $P-\{a\}$, we need only show that $Q^{\prime}$ is a retract of $P-\{a\}$ in order to conclude that $Q^{\prime}$, and so $Q$, is dismantlable. Let a mapping $f^{\prime}$ of $P-\{a\}$ to $P-\{a\}$ be given by

$$
f^{\prime}(z)= \begin{cases}f(z) & \text { if } f(z) \neq a \\ a^{*} & \text { if } f(z)=a .\end{cases}
$$

Then $f(P-\{a\})=Q^{\prime}$ and, since $f\left(a^{*}\right)=a, f^{\prime} \mid Q^{\prime}$ is the identity mapping on $Q^{\prime}$. It is straightforward to show that $f^{\prime}$ is order-preserving.

Proof of Theorem 3. We proceed by induction on $|P|$. Let $f$ be an orderpreserving mapping of $P$ to $P$ such that $Q=\{x \in P \mid f(x)=x\}$. As in the proof of Proposition 1 , there is a positive integer $n$ such that $f^{n}(P)$ is a retract of $P$. 
Observe that $Q \subseteq f^{n}(P)$ and, by Lemma $5, f^{n}(P)$ is dismantlable. If $f$ is not an automorphism of $P$ then $\left|f^{n}(P)\right|<|P|$ and, by induction, $Q$ is dismantlable. Therefore, $f$ is an automorphism of $P$.

Suppose that $a \in I(P)$ and $a \notin Q$. Since $f$ is an automorphism and $P$ is finite, $f^{i}(a) \in I(P)$; in fact, since $f^{i}(a)$ is non-comparable with $f^{i}(a)$ for $f^{i}(a) \neq f^{i}(a)$, $f^{i}(a) \in I\left(P-\left\{a, f(a), \ldots, f^{i-1}(a)\right\}\right)$, for $i=0,1, \ldots$ By Lemma $4, \quad P^{\prime}=$ $P-\left\{a, f(a), f^{2}(a), \ldots\right\}$ is dismantlable. Again, the induction hypothesis applied to $P^{\prime}$ yields that $Q$ is dismantlable. Therefore, we may assume that $I(P) \subseteq Q$. Let $a \in I(P)$ and let $a_{*}$ be the unique lower cover of $a$ in $P$. Since $f$ is an automorphism, $f\left(a_{*}\right)<f(a)=a$; it follows that $a_{*} \in Q$. Let $P^{\prime \prime}=P-\{a\}$ and let $f^{\prime \prime}=f \mid P^{\prime \prime}$. Then $f^{\prime \prime}$ is an automorphism of $P^{\prime \prime}$ and $\left\{x \in P^{\prime \prime} \mid f^{\prime \prime}(x)=x\right\}=Q-\{a\}$. We have that $P^{\prime \prime}$ is dismantlable, whence by the induction hypothesis, $Q-\{a\}$ is dismantlable. Since $a_{*}$ is the unique lower cover of $a$ in $P$ and $a_{*} \in Q$, $a \in I(Q)$. Therefore, $Q$ is dismantlable.

This work was supported in part by N.R.C. grant No. A4077.

\section{REFERENCES}

1. K. Baclawski and A. Björner, Fixed points in partially ordered sets, Advances in Math. 31 (1979) 263-287.

2. G. Birkhoff, Generalized arithmetic, Duke Math. J. 3 (1937), 283-302.

3. A. C. Davis, A characterization of complete lattices, Pacific J. Math. 5 (1955), 311-319.

4. D. Duffus and I. Rival, Crowns in dismantlable partially ordered sets, Combinatorics (Proc. Colloq. Kesthely, 1976), Colloq. Math. Jănos Bolyai 18 (1976) 271-292.

5. D. Duffus and I. Rival, Retracts of partially ordered sets, J. Australian Math. Soc. (to appear).

6. D. Duffus, I. Rival, and M. Simonovits, Spanning retracts of a partially ordered set, Research Paper No. 396, May, 1978.

7. H. Höft and M. Höft, Some fixed point theorems for partially ordered sets, Canad. J. Math. 28 (1976), 992-997.

8. I. Rival, A fixed point theorem for finite partially ordered sets, J. Comb. Th. 21 (1976), 309-318.

9. A. Tarski, A lattice-theoretical fixpoint theorem and its applications, Pacific J. Math. 5 (1955), 285-309.

DePartment of Mathematics And Statistics

THE University OF CALGARY

Calgary, Alberta T2N 1N4

TEChNische Hochschule DARMSTADT

Darmstadt, Federal Republic of Germany 